Correspondence

\title{
Updated treatment thresholds in the 2019 ESC/EAS dyslipidaemia guidelines substantially expand indications for statin use for primary prevention at population level: Results from the Rotterdam Study
}

\section{A R T I C L E I N F O}

\section{Keywords:}

Cardiovascular disease

Primary prevention

Statin

Evidence

Dyslipidaemia guidelines

Epidemiology

To the Editor,

In the recently published update of the European Society of Cardiology/European Atherosclerosis Society (ESC/EAS) clinical practice guidelines for the management of dyslipidaemias, indications for lipid-lowering treatment have been expanded to those at lower cardiovascular risk and with lower LDL levels, as well as to those 65-75 years of age, according to their level of predicted cardiovascular disease (CVD) risk $[1,2]$. To what extent these updates affect treatment recommendations at population level is unclear.

\section{Methods}

We compared the implications of the 2016 and 2019 ESC/EAS guidelines in 7262 participants free of CVD, aged 45-75 years, from the Dutch prospective population-based Rotterdam Study. Details regarding the study design, measurements and follow-up have been described in detail previously [3-5].

We calculated 10-year risk of CVD mortality following the 2016 and 2019 ESC/EAS guidelines using sex-specific Systematic Coronary Risk Evaluation (SCORE) equations for low-risk countries, and subsequently classified individuals based on whether they would qualify for lipidlowering treatment. Treatment categories were defined as per ESC/EAS guideline recommendations as either: (1) no treatment - provide lifestyle advice, (2) treatment considered - provide lifestyle intervention, consider adding drug, and (3) treatment recommended - provide lifestyle intervention and concomitant drug intervention.

\section{Results}

Mean (SD) age was 61.1 (6.9) years and $58.2 \%$ were women. Diabetes was present in $8.4 \%$ of the participants, $24.7 \%$ were smokers, mean LDL was 3.7 (0.9) $\mathrm{mmol} / \mathrm{L}$, and mean blood pressure was 137/80 (20/11) mmHg.
Besides lifestyle interventions, the 2016 and 2019 ESC/EAS guidelines recommend statin treatment in $13.2 \%(\mathrm{~N}=961)$ and $37.7 \%$ $(\mathrm{N}=2738)$ of the population, and consider statin treatment for an additional $37.0 \%(\mathrm{~N}=2689)$ and $51.4 \%(\mathrm{~N}=3733)$, respectively. When stratified by 5 -year age groups, the proportion of individuals eligible for statin treatment increases with age for both 2016 and 2019 ESC/EAS guidelines in a similar pattern (Fig. 1). However, the 2016 and $2019 \mathrm{ESC} / \mathrm{EAS}$ guidelines overall suggest a respective $29.7 \%(\mathrm{~N}=411)$ and $60.7 \%(\mathrm{~N}=839)$ of adults aged $45-55$ years to be considered for statin treatment if LDL remains uncontrolled after lifestyle interventions. Adults with an estimated 10-year SCORE risk of fatal CVD $<1 \%$ and LDL $<4.9 \mathrm{mmol} / \mathrm{L}$ were deemed to be at sufficiently low risk in the 2016 ESC/EAS guidelines and hence were suggested only lifestyle advice to modify their cardiovascular risk (evidence class/level: I/C) [2]. In the 2019 update, adults with SCORE risk $<1 \%$ and LDL between 3.0 and $4.9 \mathrm{mmol} / \mathrm{L}$ should also be considered for statin treatment (IIa/A) [1]. This led to the consideration of statin treatment in an additional 517 individuals (i.e. $37.4 \%$ of 1383 adults aged $45-55$ years) in our study.

The 2016 ESC/EAS guidelines did not provide treatment recommendations for individuals aged $>65$ years, while the 2019 ESC/ EAS guidelines recommend statin treatment in 69.2\% $(\mathrm{N}=1430)$ and consider treatment in $27.7 \%(\mathrm{~N}=571)$ among the study population aged 65-75 years $(\mathrm{N}=2065)$ (Fig. 1).

\section{Discussion}

The 2019 ESC/EAS dyslipidaemia guidelines expand treatment recommendations to asymptomatic adults with SCORE risk $<1 \%$ and LDL cholesterol levels below $4.9 \mathrm{mmol} / \mathrm{L}$, thus deeming an additional $37.4 \%$ of all individuals aged $45-55$ years to be at sufficient risk to be considered for statin treatment. This is relevant, as an additional one third of the middle-aged population should now have a discussion with their health care provider regarding initiation of statin treatment. The

DOI of original article: https://doi.org/10.1016/j.atherosclerosis.2019.08.014 


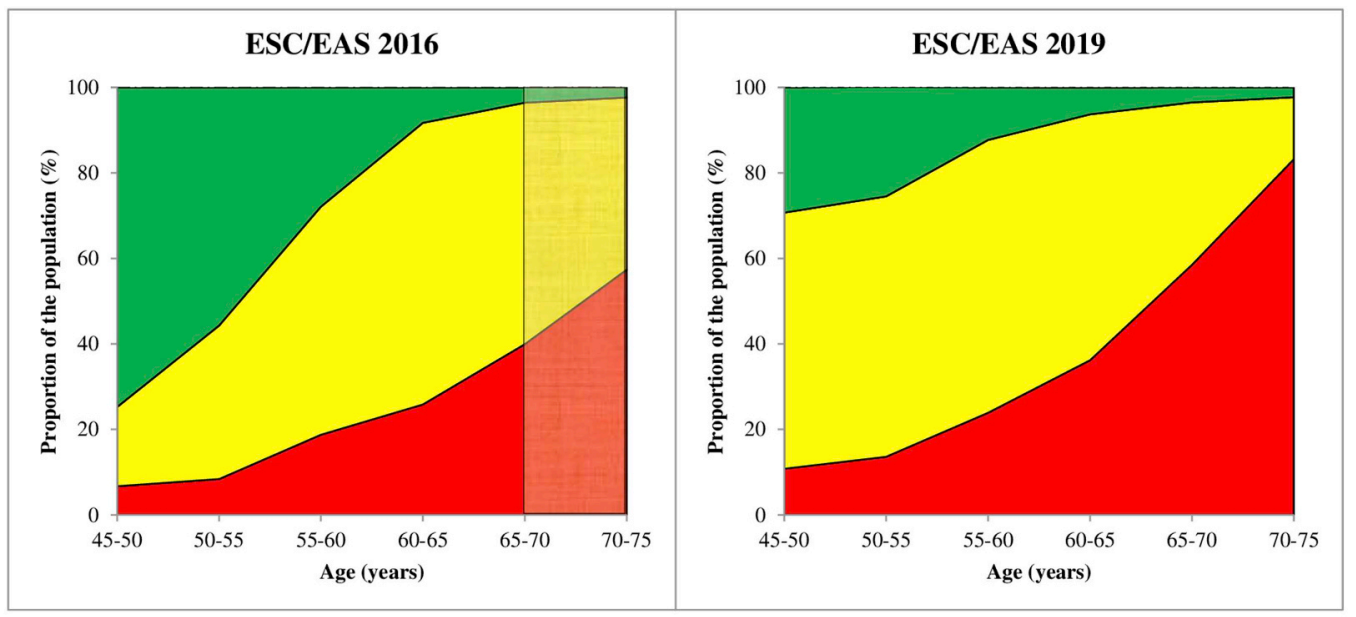

\author{
Figure Legend \\ No treatment - Lifestyle advice. \\ $\square$ Treatment considered - Lifestyle intervention, consider adding drug if uncontrolled. \\ $\square$ Treatment recommended - Lifestyle intervention and concomitant drug intervention. \\ $\square$ The 2016 ESC/EAS dyslipidaemia guidelines provided no definite lipid-lowering treatment recommendations for individuals $\geq 65$ \\ years, while the 2019 ESC/EAS guidelines expanded recommendations to individuals 65-75 years of age.
}

Fig. 1. Differences at population level in treatment recommendations for lipid-lowering treatment in primary prevention according to the 2016 and 2019 ESC/EAS dyslipidaemia clinical practice guidelines.

updates in the most recent set of guidelines can be supported by evidence that statin treatment reduces risk of hard CVD end points up to 20 years later [6], and that even younger and relatively low-risk individuals are likely to benefit from long-term statin use [7].

Moreover, following the 2019 ESC/EAS guidelines, $69.2 \%$ of asymptomatic individuals aged 65-75 years are now eligible for statin treatment in our study, and another $27.7 \%$ should be considered for statin treatment. SCORE has been shown to overestimate risk in adults over 65 years of age [8], and ESC/EAS guideline makers in 2016 noted that there was not sufficient evidence at that time to provide recommendation for statin treatment for this age group [2]. Nevertheless, expanding treatment recommendations in the 2019 ESC/EAS update to adults up to 75 years of age is an important change, supported by recent meta-analyses of randomized trials demonstrating the benefit of statin use in a primary prevention setting among older individuals [9].

Whether the broadening of statin indications in asymptomatic lowrisk individuals and those aged 65-75 years will lead to a greater uptake of primary prevention efforts and thereby a long-term lowering of the burden of CVD in Europe, remains to be investigated.

\section{Ethics statement}

The Rotterdam Study has been approved by the Medical Ethics Committee of the Erasmus MC (registration number MEC 02.1015) and by the Dutch Ministry of Health, Welfare and Sport (Population Screening Act WBO, license number 1071272-159521-PG). The Rotterdam Study has been entered into the Netherlands National Trial Register (NTR; www.trialregister.nl) and into the WHO International Clinical Trials Registry Platform (ICTRP; www.who.int/ictrp/network/ primary/en/) under shared catalogue number NTR6831. All participants provided written informed consent to participate in the study and to have their information obtained from treating physicians.

\section{Funding}

The Rotterdam Study is supported by the Erasmus MC and Erasmus University Rotterdam; the Netherlands Organisation for Scientific Research; the Netherlands Organisation for Health Research and
Development; the Research Institute for Diseases in the Elderly; the Netherlands Genomics Initiative; the Ministry of Education, Culture, and Science; the Ministry of Health, Welfare, and Sports; the European Commission (DG XII); and the Municipality of Rotterdam. Dr Pavlović is supported a scholarship from Erasmus Mundus Western Balkans, a project funded by the European Commission.

\section{Declaration of competing interest}

The authors declared they do not have anything to disclose regarding conflict of interest with respect to this manuscript.

\section{Acknowledgments}

The dedication, commitment, and contribution of inhabitants, general practitioners, and pharmacists to the Rotterdam Study are gratefully acknowledged.

\section{References}

[1] F. Mach, C. Baigent, A.L. Catapano, K.C. Koskinas, M. Casula, et al., ESC/EAS guidelines for the management of dyslipidaemias: lipid modification to reduce cardiovascular risk, Atherosclerosis 290 (2019) 140-205 2019.

[2] A.L. Catapano, I. Graham, G. De Backer, O. Wiklund, M.J. Chapman, et al., ESC/EAS guidelines for the management of dyslipidaemias, Atherosclerosis 253 (2016) 281-3442016.

[3] J. Pavlović, P. Greenland, J.W. Deckers, J.J. Brugts, M. Kavousi, et al., Comparison of ACC/AHA and ESC guideline recommendations following trial evidence for statin use in primary prevention of cardiovascular disease: results from the population-based Rotterdam study, JAMA Cardiol 1 (2016) 708-713.

[4] M.A. Ikram, G.G.O. Brusselle, S.D. Murad, C.M. van Duijn, O.H. Franco, et al., The Rotterdam study: 2018 update on objectives, design and main results, Eur. J. Epidemiol. 32 (2017) 807-850.

[5] A. Hofman, C.M. van Duijn, O.H. Franco, M.A. Ikram, H.L.A. Janssen, et al., The Rotterdam study: 2012 objectives and design update, Eur. J. Epidemiol. 26 (8) (2011) 657.

[6] I. Ford, H. Murray, C. McCowan, C.J. Packard, Long-term safety and efficacy of lowering low-density lipoprotein cholesterol with statin therapy, Circulation 133 (11) (2016) 1073-1080.

[7] Cholesterol Treatment Trialists' (CTT) Collaborators, The effects of lowering LDL cholesterol with statin therapy in people at low risk of vascular disease: meta-analysis of individual data from 27 randomised trials, Lancet 380 (2012) 581-590.

[8] M.T. Cooney, R. Selmer, A. Lindman, A. Tverdal, A. Menotti, et al., Cardiovascular risk estimation in older persons: SCORE O.P, Eur. J. Prev. Cardiol. 23 (10) (2016) 
1093-1103.

[9] Cholesterol Treatment Trialists' (CTT) Collaborators, Efficacy and safety of statin therapy in older people: a meta-analysis of individual participant data from 28 randomised controlled trials, Lancet 393 (10170) (2019) 407-415.

Jelena Pavlović, Maryam Kavousi Department of Epidemiology, Erasmus MC - University Medical Center Rotterdam, Rotterdam, the Netherlands

M. Arfan Ikram

Department of Epidemiology, Erasmus MC - University Medical Center Rotterdam, Rotterdam, the Netherlands Department of Neurology, Erasmus MC - University Medical Center
Rotterdam, Rotterdam, the Netherlands Department of Radiology, Erasmus MC - University Medical Center Rotterdam, Rotterdam, the Netherlands

Maarten J.G. Leening* Department of Epidemiology, Erasmus MC - University Medical Center Rotterdam, Rotterdam, the Netherlands Department of Cardiology, Erasmus MC - University Medical Center Rotterdam, Rotterdam, the Netherlands Department of Epidemiology, Harvard T.H. Chan School of Public Health, Boston, MA, USA

E-mail address: m.leening@erasmusmc.nl.

${ }^{*}$ Corresponding author. Departments of Epidemiology and Cardiology, Erasmus MC, University Medical Center Rotterdam, P.O. Box 2040, 3000 CA, Rotterdam, the Netherlands. 